\title{
SPREADING OF WAVE-DRIVEN CURRENTS IN A TOKAMAK
}

\author{
D. W. Ignat, R. Kaita, S. C. Jardin, and M. Okabayashi \\ Plasma Physics Laboratory \\ Princeton University \\ P. O. Box 451 \\ Princeton, New Jersey \\ USA 08543
}

ABSTRACT. Lower hybrid current drive (LHCD) in the tokamak Princeton Beta Experiment-Modification (PBX-M) is computed with a dynamic model in order to understand an actual discharge aimed at raising the central $q$ above unity. Such configurations offer advantages for steady-state operation and plasma stability. For the particular parameters of this PBX-M experiment, the calculation found singular profiles of plasma current density $J$ and safety factor $q$ developing soon after LHCD begins. Smoothing the lower hybrid-driven current and power using a diffusion-like equation and a velocity-independent diffusivity for fast-electron current brought the model into reasonable agreement with the measurements if $D_{\text {fast }} \approx 1.0 \mathrm{~m}^{2} / \mathrm{s}$. Such a value for $D_{\text {fast }}$ is in the range suggested by other work.

PACS: $52.35 . \mathrm{Fp} \quad 52.35 . \mathrm{Hr} \quad 52.40 . \mathrm{Db} \quad 52.50 . \mathrm{Gj}$

January 4, 1996

\section{DISCLAIMER}

This report was prepared as an account of work sponsored by an agency of the United States This report was prepared as an accou their Government. Neither the United States Government nor any agency thereof, nor any of their employees, makes any warranty, express or implied, or assumes any legal liability or roduct, or bility for the accuracy, completeness, or usefulness of any information, apparatus, product, or process disclosed, or represents that its use would not infringe privately owned rights. Reference herein to any specific commercial product, process, or service by trade name, trademark, manufacturer, or otherwise does not necessarily constitute or imply its endorsement, recommendation, or favoring by the United States Government or any agency thereof. The views and opinions of authors expressed herein do not necessarily state or reflect those of the United States Government or any agency thereof. 


\section{DISCLAIMER}

Portions of this document may be illegible in electronic image products. Images are produced from the best available original document. 


\section{INTRODUCTION}

The aim of the Princeton Beta ExperimentModification (PBX-M) is to explore advanced tokamak regimes with methods applicable to steady-state discharges. The term 'advanced tokamak' refers to configurations having both high bootstrap current [1] and current peaked off axis for equilibrium and stability at high $\beta[2-6]$. The ability to optimize performance by adjusting profiles of pressure and current through changes to heating and current drive power is presumed to be necessary, and the demonstration of such adjustments was a principal goal of the PBX-M [7].

We discuss here one near-term objective of the PBX-M: to demonstrate that lower hybrid current drive (LHCD) is a practical means for increasing the central safety factor $q(0)$, reversing central the magnetic shear [8], and broadening the current profile [9]. The PBX-M project can also stabilize kink modes with a close-fitting, high-conductivity shell, and modify the pressure profile with ion Bernstein wave heating [10]. Those capabilities are discussed elsewhere.

Reversed shear configurations have received considerable attention recently as a preferred concept for obtaining a steady state tokamak configuration, and improving confinement. Several large tokamaks have achieved strong reversed shear and improved energy and particle confinement with neutral beam heating and transient operational techniques $[11,12]$.

The PBX-M experimental results, given in a companion paper [13], employ LHCD to place current off the magnetic axis, the motional Stark effect (MSE) to measure the poloidal magnetic field on a central chord of the plasma [14], and a computational reconstruction of the non-circular equilibrium from MSE and surface magnetic measurements [15]. In summary, $\mathrm{PBX}-\mathrm{M}$ finds that $300 \mathrm{~kW}$ of LHCD causes $q(0)$ to rise above unity, and the current profile to broaden, but not to the extent of achieving a local maximum in current density off-axis, or achieving a reversal of shear. See figures 7 and 8 of reference (13).

\section{THE MODEL}

\subsection{TSC/LSC without Diffusion}

The Tokamak Simulation Code [16] (TSC) coupled to the Lower Hybrid Simulation Code [17] (LSC) was used to simulate the LHCD results on PBX-
M. As documented in reference (17), the program assumes an axisymmetric toroidal geometry, and uses ray tracing to determine the lower hybrid wave propagation. The influence of the local electric field on the electron velocity distribution function is neglected in the calculation of the absorbed radio frequency (rf) power. Wave-particle interactions are computed only in a direction parallel to the magnetic field, but twodimensional effects of the inductive, toroidal electric field $\left(E_{d c}\right)$ on the current are included from fits to separate Fokker-Planck calculations [18]. The resonant electron slowing-down time is considered to be much less than either the diffusion time or the acceleration time in the $E_{d c}$.

In application to PBX-M the model is usually dominated by several facts:

1. the plasmas have electron temperatures in the 1 $\mathrm{keV}$ range, while parallel index of refraction of the launched LHCD spectrum $n_{\|}$is near the relatively low value of 2 , which is nominally resonant with much hotter $(\approx 10 \mathrm{keV})$ electrons;

2. toroidal effects raise the average $n_{\|}$of the spectrum after many reflections of LHCD energy from the wall;

3. the rise in $n_{\|}$tends to be a stronger effect as density and poloidal field inside the plasma increase;

4. the toroidal electric field can have a strong influence on the rf-driven current.

These effects are well established in the literature [20$24,17]$.

Of particular importance to our case is the approximate relationship $[21,22,24]$ between $n_{\| 0}$, the the initial $n_{\|}$of a ray when launched, and $n_{\| \max }$, the maximum possible value at a particular point in the plasma:

$n_{\| \max } \lesssim n_{\| \mathbf{0}} \times \frac{R_{0} / R}{1-\left(B_{p} / B_{\phi}\right)\left(\omega_{p e} / \omega\right)}$.

Here $R_{0}$ is the initial major radius of the LHCD ray of frequency $\omega$, and $R, B_{p}, B_{\phi}, \omega_{p e}$ are the major radius, poloidal and toroidal magnetic field, and plasma frequency at some point of interest. It has been shown [24] that this maximum tends to be reached in bean-shaped plasmas such as PBX-M after many reflections from the wall, whereas it may not be reached in similar circular plasmas.

PBX-M parameters such as phasing of the LHCD antenna $\left(90^{\circ}\right)$, power transmitted, density evolution, 
neutral beam power and timing, etc., were transfered to TSC/LSC in a straightforward manner. This included $300 \mathrm{~kW}$ of LHCD applied for $250 \mathrm{~ms}$, followed by a comparable level of neutral beam heating for $200 \mathrm{~ms}$. For this experiment the central $q$ with LHCD reached a value slightly above 1.1 [25]. Assuming the full LHCD power of $300 \mathrm{~kW}$, the calculated $q(0)$ increased beyond the measurement, the $q$-profile developed a local minimum and a local maximum in the mid-radius region of the plasma, and the calculation eventually broke down owing to the singular nature of the current profile in the plasma.

A reasonable computed behavior of $q$ in space and time could be obtained by trial-and-error reductions of the rf power level, with the required reduction fraction being a function of time. A reason for this to be a legitimate procedure might be a change in efficiency or coupling to internal waves with time. Such a procedure was of course arbitrary and time-consuming.

In looking at the time evolution of current density and loop voltage, three effects appeared to be important to the creation of the singular profiles. First, the rf-driven current was deposited in a narrow portion of the cross section, consistent with Eq. (1) which suggests that the largest upshift is where the product of $B_{p}$ and square root of electron density maximizes - in other words, not near the magnetic axis and not near the plasma edge. Second, the deposition of significant rf-driven current at mid-radius pulls current density, and poloidal field, out of the center on the inductive time scale, which then causes the upshift of $n_{\|}$to be reduced at more central regions. This in turn causes the rf-driven current to move outward, and to become narrower in space.

Third, and perhaps most important, the rf heating off-axis amplifies the ohmic current there, while the ohmic current at the center of the plasma falls because of the reduced loop voltage and central cooling.

The evolution of events is illustrated in Fig. (1). Singular profiles of $q$ and toroidal current $J$ are seen in Fig. (1b) and Fig. (1c), respectively. Graphs run to 0.38 seconds, and the calculation fails owing to nonconvergence of the equilibrium shortly thereafter.

The singularities appearing in the computational model suggest application of broadening mechanisms to bring the results in agreement with experiment. At the same time, experimental profiles of the bremsstrahlung emission from the fast electrons generated during LHCD showed evidence for finite fast electron diffusion coefficient [19] in the range of 0.5 to

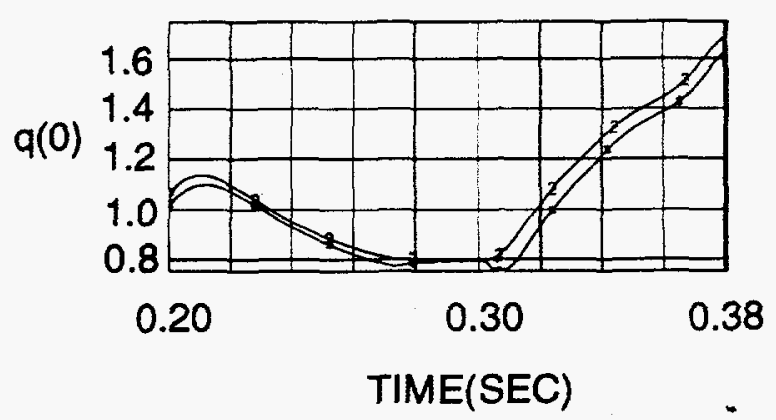

FIG. 1. (a) Central $q$ versus time, until the calculation fails. Asterisks denote the innermost computational flux surface, and the numerals ' 2 ' denote the second such flux surface. The similarity of the curves shows that the exact central $q$ is approximated satisfactorily, while the difference in the two curves is a measure of the magnetic shear at the center. The transients from starting the discharge give the behavior of $g(0)$ from $0.20 \mathrm{sec}$ to $0.27 \mathrm{sec}$; the sawtooth model in TSC keeps $q(0) \approx 0.8$ until the effects of the $L H C D$ are felt.

FIG. 1. Results of a calculation which fails owing to a pathological current profile caused by extreme localization of the rf-driven current.

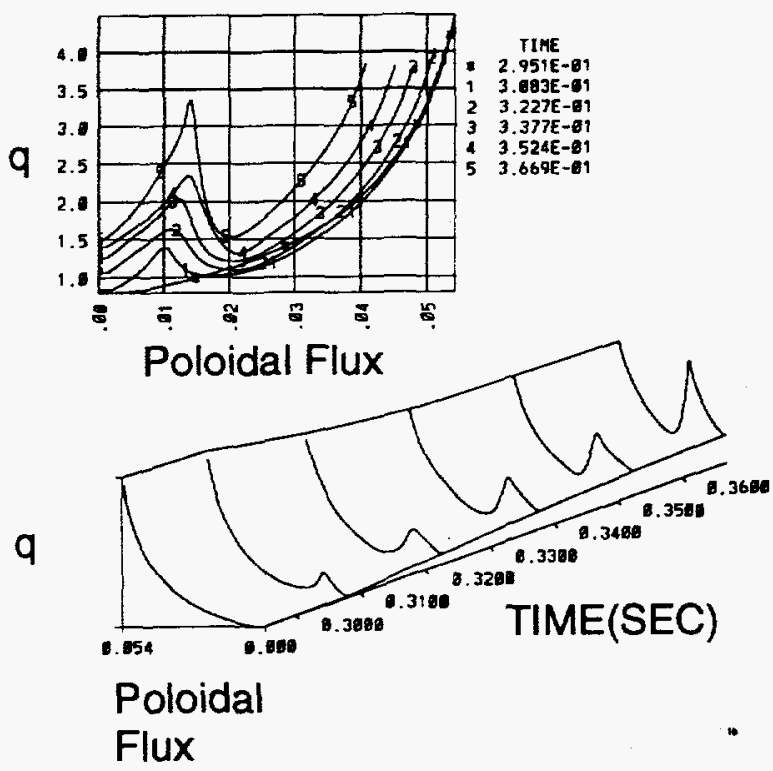

FIG. 1. (b) Profile of $q$ versus poloidal flux (given in weber per radian) as a time sequence for the same calculation, with fux increasing to the right in the 2 dimensional plot showing six times on one grid, and with fux increasing to the left on the 3-D representation. 


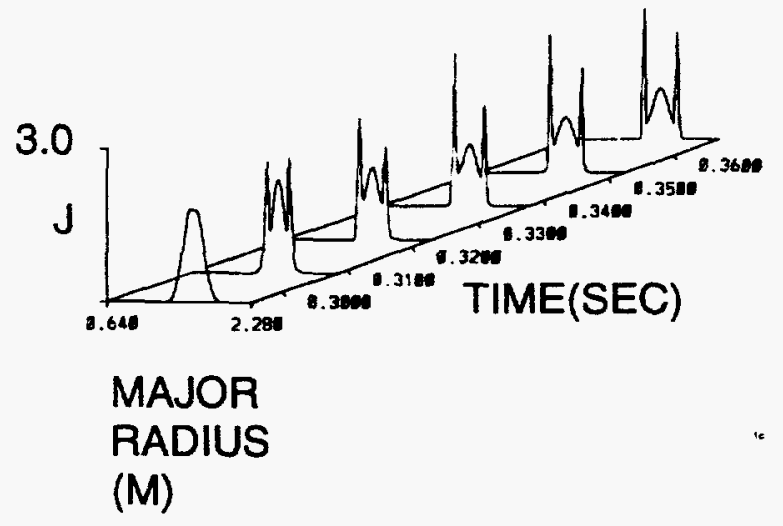

FIG. 1. (c) Spatial profile of the toraidal component of the current density $J$ (given as $\mu_{0} J$ so units are Tesla/meter) on the mid-plane of the plasma.

$2 \mathrm{~m}^{2} / \mathrm{s}$. While rapid current diffusion, heat pinches, or similar effects could have been exercised in TSC, we chose to investigate current diffusion and broadening of $\mathrm{rf}$ power in the LSC portion of the code.

\subsection{TSC/LSC with Diffusion}

\subsubsection{Heuristic Current Diffusion Estimate}

In the current diffusion model, cross-field diffusion described by a diffusivity $D_{\text {fast }}$ competes with slowingdown of the fast electrons characterized by a collision frequency $\nu$. The balance of diffusive and slowing effects determines a new rf-driven current which is altered from the rf-driven current ordinarily computed. The $\nu$ is evaluated at the local density and at a typical wave velocity. The $D_{\text {fast }}$ is an input that is varied to fit the magnitude and time behavior of the experimental plasma current and $q$ profiles. While the underlying idea is similar to the approach of other simulation codes $[26,27,23]$ which include of current diffusion, the computational method in TSC/LSC differs in detail, and is therefore described in the following.

It is possible to write the Fokker-Planck equation for the electron distribution function as [17]

$$
\begin{aligned}
\frac{\partial f}{\partial t} & =\frac{\partial}{\partial v}\left(D_{q} \frac{\partial f}{\partial v}\right)+\frac{\partial}{\partial v}\left(D_{c} \frac{\partial f}{\partial v}\right)+\frac{\partial}{\partial v}(\nu v f) \\
& +\frac{\partial}{\partial x}\left(D_{\text {fast }} \frac{\partial f}{\partial x}\right)
\end{aligned}
$$

where $f$ is the electron distribution function, and the terms on the right-hand side represent the quasilinear rf source, collisional spreading in velocity, collisional slowing, and spatial diffusion. Suppose $D_{\text {fast }}$ is independent of velocity, or that the range of velocities of interest is small if $D_{\text {fast }}$ is velocity-dependent. On multiplication by the electric charge and velocity and integration over velocity, one obtains

$$
\begin{aligned}
\frac{\partial J}{\partial t} & =-e \int D_{q} \frac{\partial f}{\partial v} d v-e \int D_{c} \frac{\partial f}{\partial v} d v-e \int \nu v f d v \\
& +\frac{\partial}{\partial x}\left(D_{\text {fast }} \frac{\partial J}{\partial x}\right)
\end{aligned}
$$

The collisional diffusion in the second term on the right hand side is small compared to the slowing down effect in the third term in the above-thermal velocity region of interest for current drive. Therefore, the second term can be ignored. If the range of velocities of current-carrying electrons is small, then $\nu$ can be treated as independent of velocity, and the third term simply becomes $-\nu J$.

Now we come to the first term, $-e \int D_{q} \partial f / \partial v d v$. The units are current density per time, and the physical origin is an rf-driven current. In the absence of diffusion, $D_{\text {fast }}=0$, this flux in velocity would be balanced against slowing down characterized by the collision rate $\nu$. Therefore, this source term may be expressed in an approximate way as $\nu J_{0}$, where $J_{0}$ is the rf current normally calculated in TSC/LSC, and the $\nu$ is the same collisional slowing time constant given in Eq. (3). Then,

$\frac{\partial J}{\partial t}=\nu\left(J_{0}-J\right)+\frac{\partial}{\partial x}\left(D_{\text {fast }} \frac{\partial J}{\partial x}\right)$,

so that a diffusion-like equation for current density is obtained.

The derivation is heuristic rather than formal, but the result in Eq. (4) does have the desirable properties:

1. As $D_{\text {fast }}$ becomes small, $J$ keeps close to $J_{0}$, in a time scaled by the slowing of the fast electrons.

2. As $D_{\text {fast }}$ becomes large, the long term values of $J$ and $J_{0}$ differ progressively.

In Eq. (4) the spatial derivatives on $J$ are written for Cartesian coordinates. In the actual calculations, the one space-like variable is normalized poloidal flux $\hat{x}$, taken proportional to the square of the average radius of the flux surface. Since our simulations are concerned with times long compared to the slowing down time, we set the time derivative to zero. The equation is then

$\frac{d}{d \hat{x}}\left(\hat{x} D_{\text {fast }}(\hat{x}) \frac{d J(\hat{x})}{d \hat{x}}\right)=\frac{a^{2} \nu(\hat{x})}{4}\left(J(\hat{x})-J_{0}(\hat{x})\right)$

where we have explicitly indicated the dependence of quantities on $\hat{x}$, the factor ' 4 ' is the mathematical consequence of our assumption that $\hat{x}$ is proportional to 
radius squared, and $a$ represents the average radius at the plasma boundary, where $\hat{x}=1$. Inversion of a tridiagonal matrix [32] solves this equation for $J(\hat{x})$ on a uniform grid in $\hat{x}$ after expressing the derivative term in finite difference form, and applying boundary conditions. The boundary condition on $J$ at the outer boundary is a zero value. At the inner boundary, i.e., the center, the condition of zero flux of $J$ is used.

In the present work, $D_{\text {fast }}$ is constant in $\hat{x}$ and

$\nu(\hat{x})=\ln \lambda \frac{n_{e}(\hat{x}) e^{4}}{4 \pi \epsilon_{0}^{2} m_{e}^{2} c^{3}} \times n_{\|}^{3}$

with $n_{\|}=2$. A typical value for the electron density $n_{e}$ is $3 \times 10^{19} \mathrm{~m}^{-3}$, which yields a typical value for $\nu$ of 250 per second, so that if $D_{\text {fast }} \approx 1 \mathrm{~m}^{2} / \mathrm{sec}$ then a diffusion scale length $\sqrt{D_{\text {fast }} / \nu}$ is approximately 0.07 meter.

In Eq. (6) above, $\ln \lambda$ is the Coulomb logarithm, $\epsilon_{0}$ is the permittivity of free space, $m_{e}$ and $e$ are the electron mass and charge.

\subsubsection{Heuristic Power Diffusion Estimate}

The steps in getting a current can be summarized as follows:

1. an estimate of the power deposited per unit volume $P_{0}(\hat{x})$ is computed from the ray tracing, plus quasi-linear development of an electron distribution function in the parallel velocity;

2. the $E_{d c}(\hat{x})$ and distribution function yield the undiffused current $J_{0}(\hat{x})$ through the KarneyFisch [18] prescription;

3. the diffused current $J(\hat{x})$ is found from Eq. (5);

4. the $E_{d c}(\hat{x})$ is corrected and steps 2 . and 3 . are repeated as necessary.

The quantities $J_{0}$ and $P_{0}$ appear similar in form on cursory inspection of graphs. (See for example figure 4 of reference (17).) In contrast, the diffused current density $J$ can be quite different from $J_{0}$, and therefore quite different in radial distribution from the $P_{0}$ found from ray tracing. This situation contrasts with the intuition that power density is proportional to current density times background number density. A more intuitive result can be had from an option to spread the deposited if power according to

$$
\begin{aligned}
P(\hat{x}) & =\alpha \frac{J(\hat{x}) n_{e}(\hat{x})}{\int J(\hat{x}) n_{e}(\hat{x}) d V(\hat{x})} \int P_{0} d V \\
& +(1-\alpha) P_{0}(\hat{x})
\end{aligned}
$$

Here, $P$ is the diffused power, $\alpha$ ranges from 0 (no spreading of $P_{0}$ ) to 1 (full spreading), and $d V$ is a volume element. Note that $P \propto J \times n_{e}$ for $\alpha=1$, in accordance with the current drive literature [28], whereas $P$ and $J$ have different spatial forms in general if $\alpha=0$.

\subsubsection{Discussion}

It should be plain that our model is not a simulation of energetic electron diffusion in that it does not transport fast particles or change the rf wave damping because of the motion of fast particles from one location to another. The diffusion model in itself does not pay attention to Faraday's law, although the physics of induction is treated in the TSC calculation.

It is interesting to note that the integral of $J$ over the plasma cross section, or total diffused $\mathrm{rf}$-driven current $I$, is not constrained to equal $I_{0}$, the same integral over $J_{0}$. Diffusion to the edge of the plasma does cause a loss of current, but diffusion can in some circumstances increase current by moving fast electrons to regions of lower collision rate. However, $I$ is less than $I_{0}$ for parameters encountered for PBX$M$ in this paper: deposition at mid-radius and mildly peaked density profile.

The heuristic smoothing method of some earlier references $[26,27,23]$ treats $D_{\text {fast }}$ and $\nu$ as constants, sets the change in total current from an estimate for the overall effect of current diffusion, and finds the value needed for $D_{\text {fast }}$ as an eigenvalue from iterative shooting solutions of an equation equivalent to our Eq. (5).

As far as we understand, spreading of current in the past $[26,27]$ has not been accompanied by a spreading of the power.

It should be mentioned that other approximate algorithms for the diffusion of rf-driven current have been proposed recently $[29,30]$.

\section{SIMULATIONS WITH DIFFUSION}

\subsection{Summary of Effects of $D_{\text {fast }}$ and $\alpha$}

To test the effect of the current diffusion model in TSC/LSC, the PBX-M discharge 313258 was simulated with $D_{\text {fast }}$ having values, in $\mathrm{m}^{2} / \mathrm{s}$, as follows: 0.0 (results given in Fig. 1 ) $; 0.1 ; 0.3 ; 1.0 ;$ and 3.0 . Good results in terms of matching data was obtained with $D_{\text {fast }}=1.0$ and with full spreading of the power deposited, that is, with $\alpha=1$ in Eq. (7). Results are 
shown in Fig. (2).

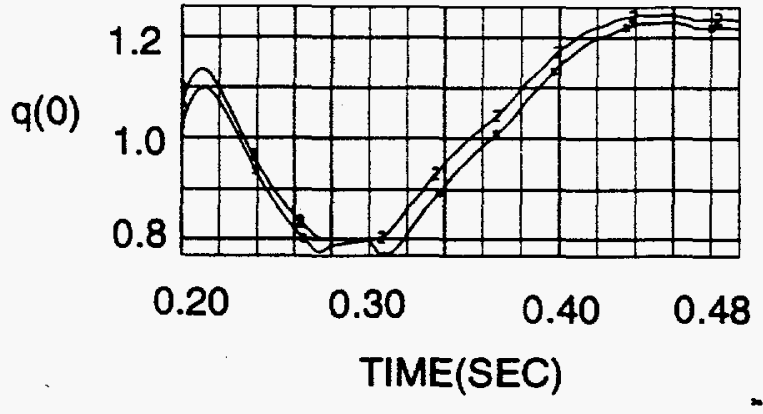

FIG. 2. (a) Central $q$ versus time. Behavior prior to the application of $L H C D$ at $0.3 \mathrm{sec}$ is the same as in other figures.

FIG. 2. Results of a successful calculation in which current and power are diffused: $D_{\text {fast }}=1 \mathrm{~m}^{2} / \mathrm{sec} ; \alpha=1$.
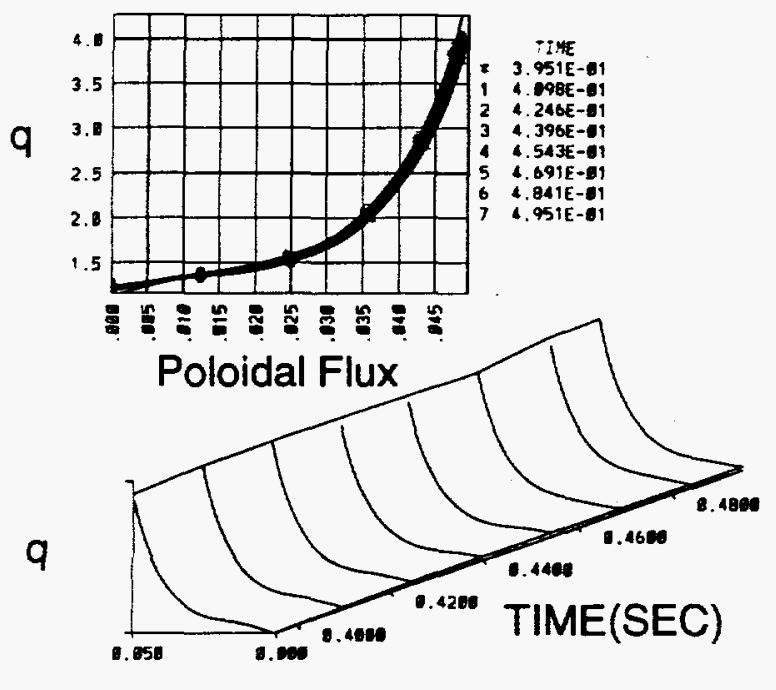

Poloidal

Flux

FIG. 2. (b) Profile of $q$ versus flux as a time sequence.

Note that while $q(0)$ rises to a value slightly above 1.2 , the overall appearance of the $q$-profile does not show a region of reversed shear. We feel this simulation is consistent with the measurements; it is obviously far more consistent with measurements than the results of Fig. (1).

Results with no power spreading ( $\alpha=0)$, but still with current diffusion, are given in Fig. (3). The central $q$, which reaches 1.3 , with no power spreading is slightly more than the value with power spreading. This is consistent with the intuition that the reduced heating in the center means less ohmic current in the center, and thus greater $q$.

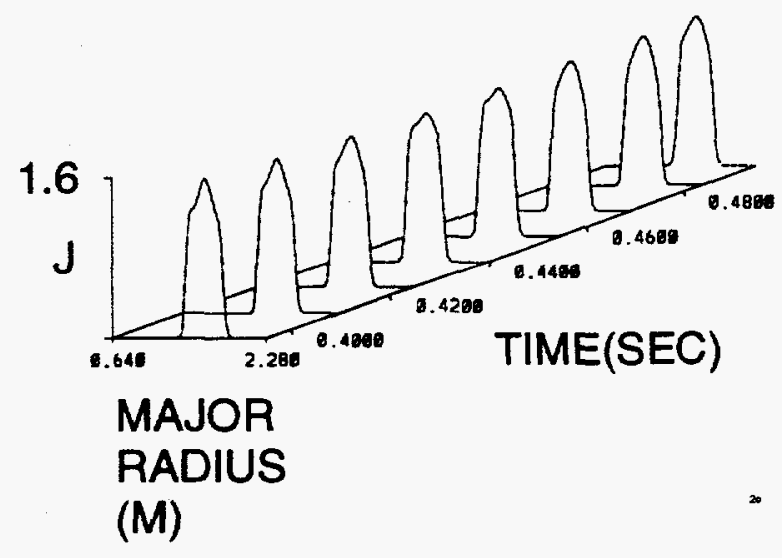

FIG. 2. (c) Spatial profile of current density for the same calculation.

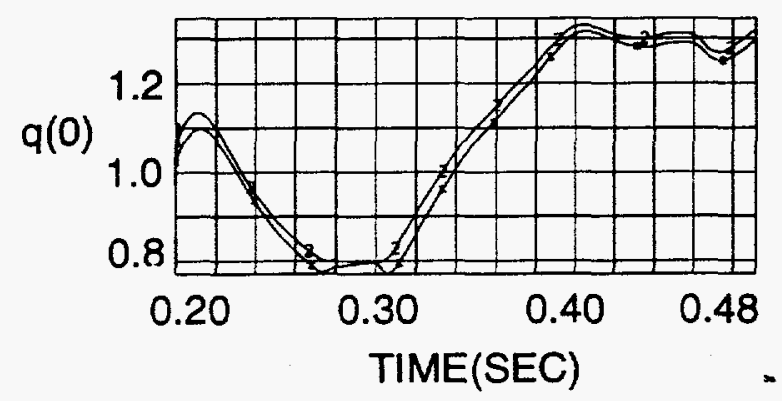

FIG. 3. (a) Central $q$ versus time.

FIG. 3. Results of a successful calculation in which current only is diffused: $D_{\text {fast }}=1 \mathrm{~m}^{2} / \mathrm{sec} ; \alpha=0$.

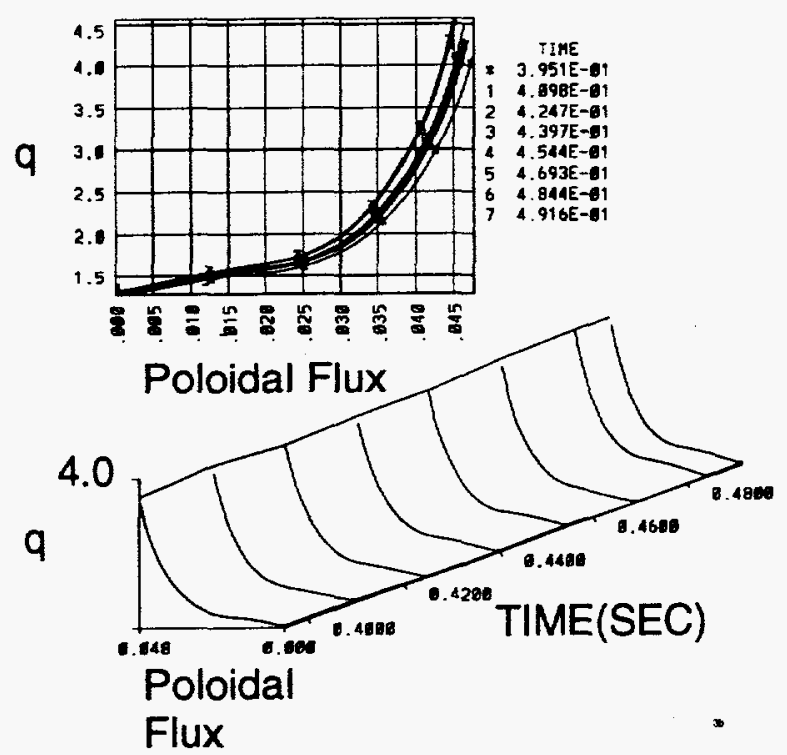

FIG. 3. (b) Spatial profile of $q$ as a time sequence. 


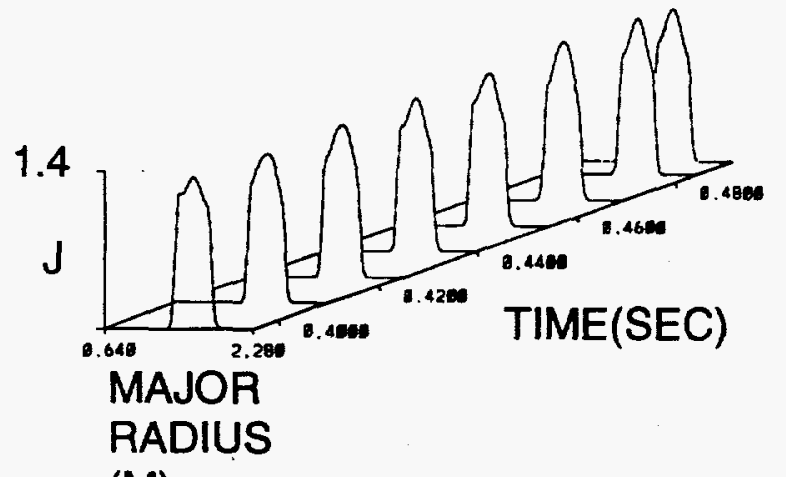

(M)

FIG. 3. (c) Spatial profile of current density for the same calculation.

The other values for $D_{\text {fast }}$ did not give a good correspondence with the data, as follows.

1. $D_{\text {fast }}=0.1$ and $D_{\text {fast }}=0.3 \mathrm{~m}^{2} / \mathrm{sec}$ and $\alpha=1$ led to a computation in which the electron temperature eventually fell so much that the lower hybrid power could not be absorbed, and the rf-driven current experienced a precipitous drop. Prior to the fall, the central $q$ did exceed unity.

2. $D_{\text {fast }}=3.0 \mathrm{~m}^{2} / \mathrm{sec}$ led to a computation showing small effects on central $q$, which never rose above unity after the LHCD started.

\subsection{Predictions at High Power}

The goal for simulations of experiments and comparisons with experimental data is twofold: understand the important effects at work; and, then, predict outcomes of future experiments.

Therefore, we now apply our model with $D_{\text {fast }}=1$ and $\alpha=1$ to a high power PBX-M experiment. If the LHCD power is increased to $1.2 \mathrm{MW}$, then TSC/LSC predicts that $q(0)$ reaches 1.8 at 0.5 seconds as shown in Fig. (4a), and that the shear reverses at a middle location in the plasma at a time around 0.45 seconds as shown in Fig. (4b). At 0.5 seconds the $q(0)$ is still rising. Figure (4c) shows, however, that the width of the current profile is stable.

This is the maximum power level available on PBX$M$, and the expected $q(0)$ exceeds the values required to access second stability or stable high beta equilibria with the indentations achievable on PBX-M [33].

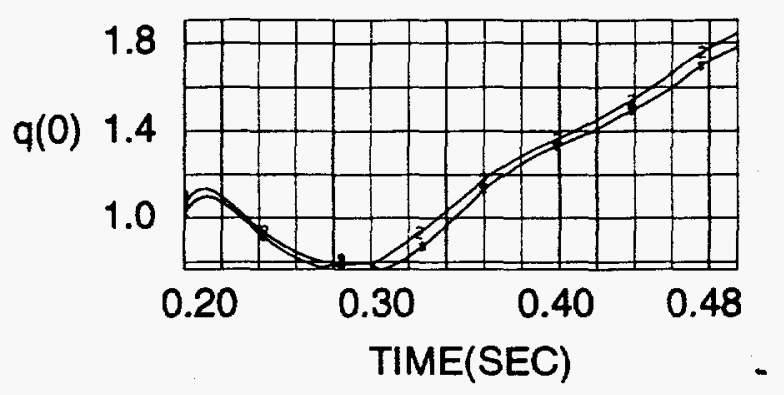

FIG. 4. (a) Central $q$ versus time.

FIG. 4. Results of a calculation in which $1.2 \mathrm{MW}$ of $L H C D$ power is applied to $P B X-M$, and a diffusion of both power and current is assumed.

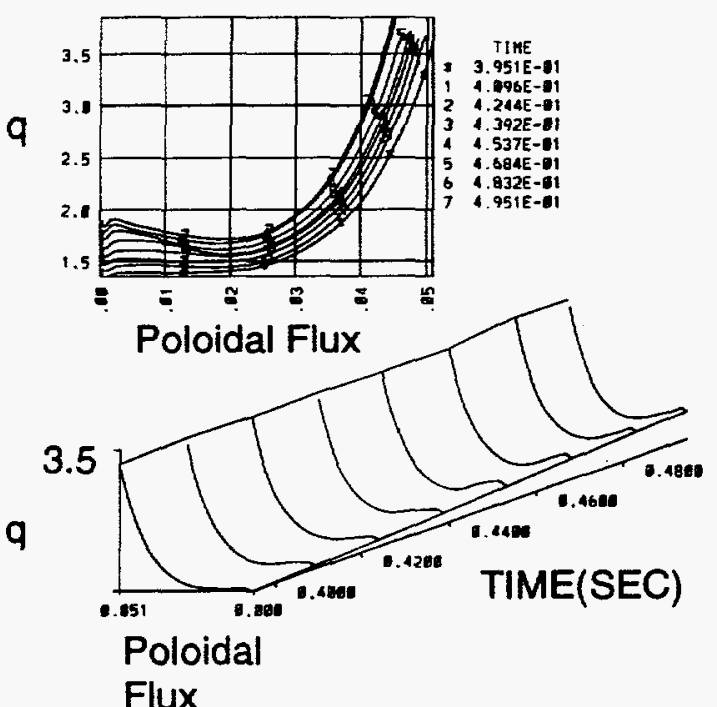

FIG. 4. (b) Spatial profile of $q$ as a time sequence. Note that there is reversed shear.

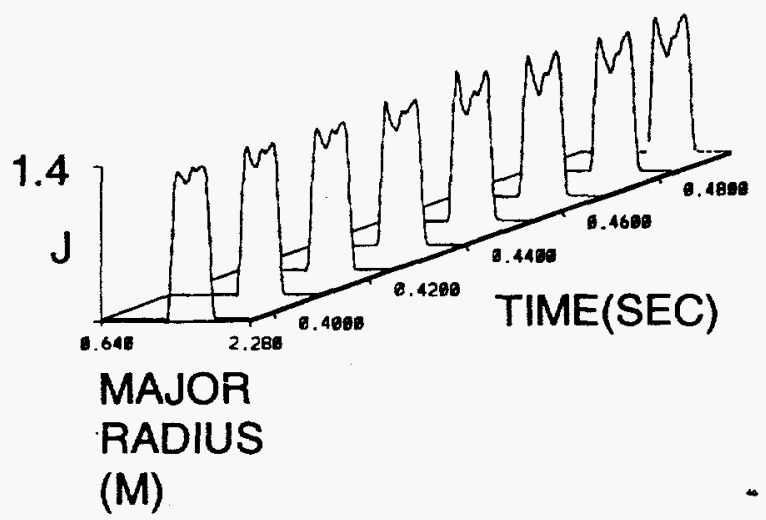

FIG. 4. (c) Spatial profile of current density for the same calculation. 


\section{SUMMARY AND CONCLUSION}

The PBX-M experiment with LHCD shows a modest rise in central $q$, but no reversal of shear. A standard computational method for LHCD shows significant reversal of shear and significant rise in $q(0)$ for the particular parameters of this experiment. Those parameters feature high density, large upshifts of $n_{\|}$, and lead to strong off-axis current drive and electron heating by the rf power. That standard computation eventually fails owing to singular current profiles.

Reasonable matching of the measured $q$ profile depended on a broadening of the rf-driven current according to a heuristic method which uses a diffusionlike equation. An accompanying broadening of the associated heating power tends to reduce the computed rise in central $q$.

The size of the diffusion coefficient needed was in the range of $1.0 \mathrm{~m}^{2} / \mathrm{sec}$, which is consistent with other findings [19]. For such a value of $D_{\text {fast }}$ a crude estimate for the diffusion scale length $\sqrt{D_{\text {fast }} / \nu}$ is $0.07 \mathrm{~m}$, to be compared with the radial scale of the tokamak, 0.30 m.

Because the model is heuristic, and diffusive effects appear to be important, it seems vital to have a more complete theory to analyze experiments in this range of parameters.

It cannot be known from the present work if an experiment-theory comparison in other ranges of parameters will behave similarly, particularly regarding the need for a large diffusion parameter to match the data. It would be specially important to know how comparisons would fare under conditions of high electron temperature $T_{e}$, well matched to the low $n_{\|}$(high phase velocity) desired for high efficiency of current drive.

Extrapolation of the present experiment to higher power shows that $q(0) \sim 1.8$ and reversed shear can be achieved on PBX-M, even under conditions of low temperature.

Our efforts at modeling this particular PBX-M discharge encountered parameters for which the equations governing absorption of LHCD power, resulting current, flux and heat diffusion, develop unstable solutions. We encountered two types of instabilities: (1) concentration of rf-driven current giving a computational problem with equilibrium; and (2) loss of sufficient electron temperature and toroidally induced upshift of the $n_{\|}$to allow absorption of LHCD power. The possibility of such unstable solutions was not anticipated prior to our calculations.

\section{ACKNOWLEDGMENTS}

This work was supported by the U.S. Department of Energy under the contract DE-AC02-76-CH03073. The present current diffusion algorithm is a descendant of a method used by V. Fuchs, who explained his approach to one of us (DWI) in valuable discussions. We thank E. J. Valeo for his interest and helpful advice. 


\section{REFERENCES}

[1] JARDIN, S.C., et al., Fus. Engineer. Design 25215 (1994).

[2] SYKES, A., et al., Phys. Rev. Lett. 39757 (1977).

[3] GREENE, J.M., CHANCE, M.S., Nucl. Fusion 21453 (1981).

[4] CHANCE, M.S., et al., in Proceedings of the Second Symposium on Plasma Dynamics: Theory and Applications,

M. Tessaroto, ed., University of Trieste, Italy, 1992; pp. 15 -25 .

[5] KESSEL, C., et al., Phys. Rev. Lett. 721212 (1994).

[6] MANICKAM, J., et al., Phys. Plasmas 11601 (1994).

[7] BELL, R.E., et al., Phys. Fluids B 21271 (1990).

[8] Magnetic shear refers to the rate of change of direction of magnetic field with respect to distance across the field. A simple and customary definition of magnetic shear is the logarithmic derivative of $q$ with respect to position $r$ : $(r / q)(d q / d r)$.

[9] BERNABEI, S., et al., Phys. Fluids B 52562 (1993).

[10] LEBLANC, B., et al., Phys. Plasmas 2741 (1995).

[11] TURNBULL, A.D., et al., Phys. Rev. Lett. 74718 (1995).

[12] LEVINTON, F.M., et al., submitted to Phys. Rev. Letters, August 1995.

[13] KAITA, R., et al., submitted for publication to this journal.

[14] LEVINTON, F.M., et al., Phys. Rev. Lett. 632060 (1989).

[15] HIRShMAN, S.P., et al., Phys. Plasmas, in press.

[16] JARDIN, S.C., et al., J. Comput. Phys. 66481 (1986).

[17] IGNAT, D.W., et al., Nucl. Fusion 34837 (1994).

[18] KARNEY, C.F.F, FISCH, N.J., Phys. Fluids 29180 (1986).

[19] JONES, S.E., et al., Plasma Phys. Control. Fusion 35 1003 (1993).

[20] BONOLI, P.T., ENGLADE, R.C., Phys. Fluids 292937 (1986).

[21] KUPFER, K., MOREAU, D. Nucl. Fusion 321845 (1992).

[22] TAKAHASHI, H., et al., in Proceedings of the Twentieth European Physical Society Conference on Controlled Fusion and Plasma Physics, (Lisbon, Portugal, 1993) III 901 (1993).

[23] KESNER, J., et al., Nucl. Fusion 34619 (1994).

[24] PAOLETTI, F. et al., Nucl. Fusion 34 771-776 (1994).

[25] KAITA, R., et al., in Proceedings of the TwentyFirst European Physical Society Conference on Controlled Fusion and Plasma Physics, Europhysics Conference Abstracts 18B, Part III, 1094 (1994).

[26] FUCHS, V., et al., Nucl. Fusion 291479 (1989).

[27] TANI, K., et al., J. Comput. Phys. 98332 (1992).

[28] FISCH, N.J.; Rev. Mod. Phys. 59175 (1987).

[29] CAIRNS, R.A., et al., Nucl. Fusion 351413 (1995).

[30] CAIRNS, R.A., et al., to be published.

[31] DEMERS, Y., et al., Proceedings of the Eleventh Topical Conference on Radio Frequency Power in Plasmas, (Palm Springs, CA, May 1995).

[32] PRESS, W.H., et al., Numerical Recipes in Fortran (Cambridge University Press, Cambridge, 1989); p40.

[33] BOL, K., et al., Princeton University, Plasma Physics Laboratory Report PPPL-2032 (September 1983) 74 pp. 